\title{
The Chiral Phase Transition from Lattice QCD with Physical Pion Masses and Domain Wall Fermions
}

\author{
Chris Schroeder (for the HotQCD collaboration)* \\ Lawrence Livermore National Laboratory \\ E-mail: schroeder10@llnl.gov
}

\begin{abstract}
The HotQCD collaboration has extended its study of the finite temperature transition in $2+1$ flavor QCD using domain wall fermions (DWF) to the physical point. With chiral fermions and truly physical pion masses, these calculations are arguably the most realistic of their kind to date, and not possible without a combination of algorithmic advances (chiefly the dislocation suppressing determinant ratio, DSDR), highly optimized BAGEL/CPS software, and access to the LLNL/IBM 20 Petaflops Sequoia Blue Gene/Q. All results are for a fixed temporal lattice size of 8, yet discretization effects, at least for the spectrum at zero temperature, are expected to be at the $5 \%$ level. Presented here are early results - in particular, the critical temperature and pion mass dependence of the light quark chiral susceptibility.
\end{abstract}

8th International Workshop on Critical Point and Onset of Deconfinement

March 11-15

Napa, California, USA

\footnotetext{
* Speaker.
} 


\section{Introduction}

The nature of the chiral symmetry breaking transition in QCD at finite temperature and zero chemical potential is of great fundamental interest and essential to understanding the results of relativistic heavy ion collision experiments and the early evolution of the universe. The transition has been studied intensely in lattice gauge theory but until now, calculations with both the physical pion mass and a chiral fermion discretization that exhibits $S U(2) \times S U(2)$ chiral symmetry at finite lattice spacing have been unfeasible due to computational cost. While studies with staggered fermions $[1,2]$ require the fourth-root trick and are afflicted with taste symmetry breaking, which necessitates a computationally demanding continuum limit in order to address true chiral physics, Wilson and twisted mass fermions break chiral symmetry entirely and cannot be used to simulate the transition with physical pions without much finer lattices and larger volumes than have been used to date. It is a marked improvement to study the transition with chiral fermions such as domain wall or overlap fermions.

Previous studies with chiral fermions $[3,4,5]$ have been feasible only with unrealistically heavy pions, but with groundbreaking computational resources and algorithmic advances along with high performance software, the HotQCD collaboration is now investigating the chiral symmetry breaking transition with domain wall fermions (DWF) at the physical pion mass. This work is an extension of HotQCD's study of the transition with domain wall fermions at a pion mass of $200 \mathrm{MeV}$ [4], and many details omitted here can be found there and in upcoming publications.

As in [4], we use the domain wall fermion action augmented by DSDR [6, 7, 8] and the Iwasaki gauge action. By steering simulations away from artifacts known as dislocations, which contribute greatly to residual chiral symmetry breaking when the extent of the algorithmic fifth dimension $L_{S}$ is finite, DSDR reduces the computational cost of these calculations by more than order of magnitude. While DSDR is the key to reaching physical pion masses at gauge couplings large enough to be feasible, we derive an additional speedup of approximately two by using the Möbius DWF formulation [9], which further reduces residual chiral symmetry breaking. For field evolution, we use the Rational Hybrid Monte Carlo (RHMC) algorithm with Sexton-Weingarten multiscale integration and force gradient forecasting.

Even with significant algorthmic advances, this work would not have been possible without access to the LLNL/IBM Sequoia Blue Gene/Q supercomputer and the highly optimized BAGEL/CPS software, together yielding sustained per-node performance roughly 20 times greater than achieved previously, i.e., on the Blue Gene/P architecture. Figure 1 illustrates the unprecedented performance of this combination of hardware and software. An excellent description of the machine and the bulk of the extensive work invested in the software can be found in [10].

\section{Results}

While many quantities can and will be computed from the ensembles produced, the focus here is on the light quark chiral condensate,

$$
\left\langle\bar{\psi}_{l} \psi_{l}\right\rangle=\frac{T}{V} \frac{\partial \ln Z}{\partial m_{l}}=\frac{1}{N_{\sigma}^{3} N_{\tau}}\left\langle\operatorname{Tr} M_{l}^{-1}\right\rangle,
$$




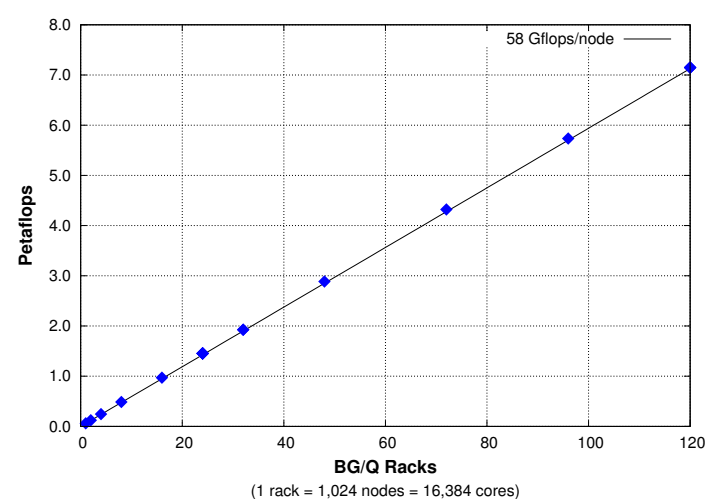

Figure 1: Weak scaling performance of the BAGEL Möbius DWF conjugate gradient solver on the LLNL/IBM Sequoia Blue Gene/Q supercomputer.

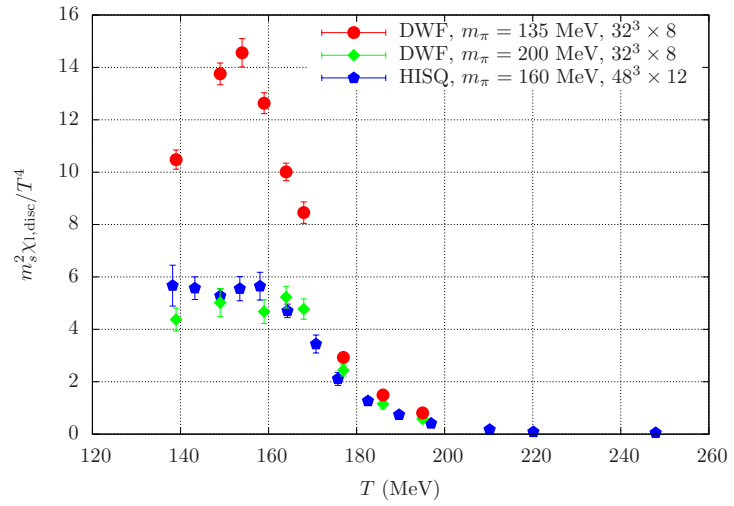

Figure 2: The disconnected chiral susceptibility for three physical pions, for three $200 \mathrm{MeV}$ pions, and for staggered (HISQ) quarks with $m_{\pi}=160 \mathrm{MeV}$.

( $N_{\sigma}$ and $N_{\tau}$ are the physical spatial and temporal extents of the lattice, and $M_{l}$ the light quark Dirac matrix) and the disconnected part of the light quark chiral susceptibility,

$$
\frac{\chi_{1, \mathrm{disc}}}{T^{2}}=N_{\sigma}^{3} N_{\tau}^{3}\left(\left\langle\left(\bar{\psi}_{l} \psi_{l}\right)^{2}\right\rangle-\left\langle\bar{\psi}_{l} \psi_{l}\right\rangle^{2}\right)
$$

which peaks at the pseudo-critical temperature and is simple to renormalize. The $\overline{\mathrm{MS}}(\mu=2 \mathrm{GeV})$ scheme is used here. The temperature dependence of this quantity, per flavor, is shown in Figure 2, along with results for DWF with $200 \mathrm{MeV}$ pions and the HISQ (staggered) action from [2] with a nominal pion mass of $160 \mathrm{MeV}$ for comparison. While the calculation is still in progress (with emphasis on the possibility of finite volume effects), there are already several prominent features. Above all, the height of the peak, a measure of condensate fluctuation at the transition temperature, is substantially greater for the lighter pions. In fact, the peak is over 33\% (75\%) higher than expected from the $200 \mathrm{MeV}$ DWF (160 MeV HISQ) result and $O(4)$ scaling, $\chi_{1, \text { disc }} \sim m_{\pi}^{-1.6}$ [11]. In addition, relative to the $200 \mathrm{MeV}$ DWF calculation, the location of the peak (i.e., the pseudo-critical temperature) shifts to significantly lower temperatures, between 150 and $160 \mathrm{MeV}$, consistent with results obtained previously using staggered fermions.

I also present very early results for the light quark chiral condensate computed at a pion mass of $100 \mathrm{MeV}$, well below the physical point. This calculation is motivated by the question of the nature of the transition at lower light quark masses. As sketched in Figure 3, it may be that the transition becomes first-order as the light quark mass is reduced below the physical point. To discover this change in the nature of the transition would be extremely interesting and may be feasible only with chiral fermions. We have begun to compute the chiral condensate at a single temperature, $160 \mathrm{MeV}$, initializing one stream with an ordered gauge field and another with a disordered field. The chiral condensates for the two streams merge without hesitation, as shown in Figure 4, an indication that there is no metastability or first-order phase transition for this pion mass, near this temperature. It is likely that $160 \mathrm{MeV}$ is too high a temperature to observe this behavior and it may also be that the light quark mass is too large to be in the region of interest. A similar calculation at a lower temperature (also with $m_{\pi}=100 \mathrm{MeV}$ ) has been commenced. 


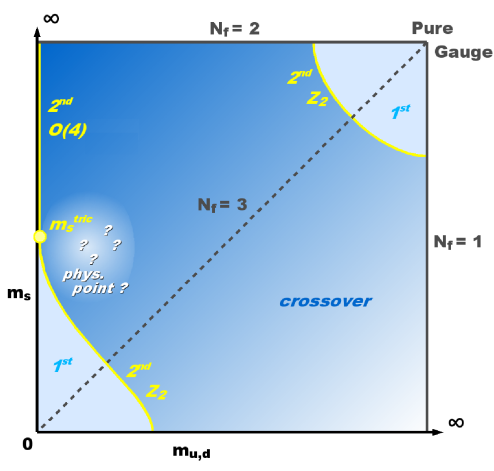

Figure 3: The anticipated dependence of the nature of the chiral phase transition on the light and strange quark masses, i.e., the "Columbia plot."

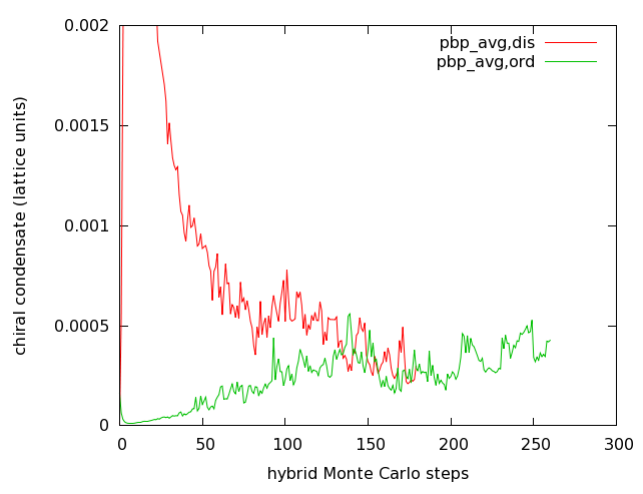

Figure 4: Evolution of the chiral condensate for $m_{\pi} \approx 100 \mathrm{MeV}$ and $T \approx 160 \mathrm{MeV}$ in simulation time for ordered and disordered initializations.

\section{Summary}

The HotQCD collaboration has made substantial progress in studying the chiral phase transition of QCD with chiral, domain wall fermions and three degenerate, physical-mass pions, but there is still much work to be done. Preliminary results for the disconnected chiral susceptibility suggest the true critical temperature is not far from that derived from previous calculations using staggered fermions; however, the fluctuations at $T_{c}$ appear to be larger than in staggered calculations and to grow more rapidly as $m_{\pi}$ decreases than $\chi \mathrm{PT}$ predicts. Finally, a study of the nature of the phase transition at sub-physical pion masses has begun and first results have been obtained for $m_{\pi} \approx 100 \mathrm{MeV}$ and $T \approx 160 \mathrm{MeV}$.

\section{References}

[1] S. Borsanyi et al. [Wuppertal-Budapest Collaboration], JHEP 1009, 073 (2010).

[2] A. Bazavov, T. Bhattacharya, M. Cheng, C. DeTar, H. T. Ding, S. Gottlieb, R. Gupta and P. Hegde et al., Phys. Rev. D 85, 054503 (2012).

[3] G. Cossu, S. Aoki, H. Fukaya, S. Hashimoto, T. Kaneko, H. Matsufuru and J. -I. Noaki, arXiv:1304.6145 [hep-lat].

[4] A. Bazavov et al. [HotQCD Collaboration], Phys. Rev. D 86, 094503 (2012).

[5] S. Borsanyi, Y. Delgado, S. Durr, Z. Fodor, S. D. Katz, S. Krieg, T. Lippert and D. Nogradi et al., Phys. Lett. B 713, 342 (2012).

[6] P. Vranas, Proceedings, NATO Advanced Research Workshop, Dubna, Russia, October 5-9, 1999, C99-10-05.3, p.11-26, 1999.

[7] P. M. Vranas, Phys. Rev. D 74, 034512 (2006).

[8] D. Renfrew, T. Blum, N. Christ, R. Mawhinney and P. Vranas, PoS LATTICE 2008, 048 (2008).

[9] R. C. Brower, H. Neff and K. Orginos, Nucl. Phys. Proc. Suppl. 153, 191 (2006).

[10] P. A. Boyle, PoS LATTICE 2012, 020 (2012).

[11] J. Engels, S. Holtmann, T. Mendes and T. Schulze, Phys. Lett. B 514, 299 (2001). 